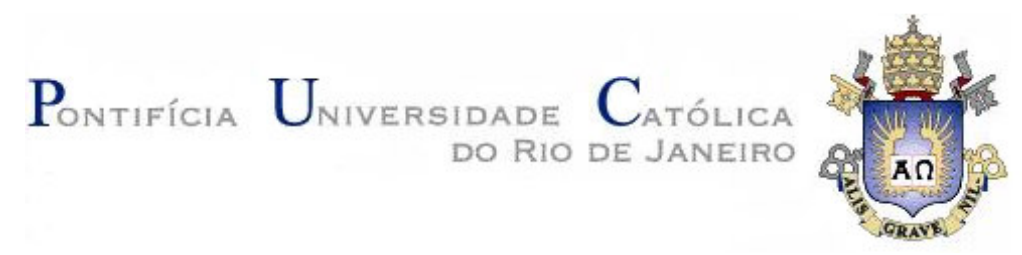

João Luiz de Oliveira Santa Rita

Estratégia de contratação de fretes: um estudo de caso na indústria do cimento

Dissertação de Mestrado

Dissertação apresentada como requisito parcial para obtenção do grau de Mestre pelo Programa de Pós-Graduação em Engenharia de Produção do Departamento de Engenharia Industrial da PUC-Rio.

Orientador: Prof. José Eugenio Leal

Rio de Janeiro

Maio de 2013 


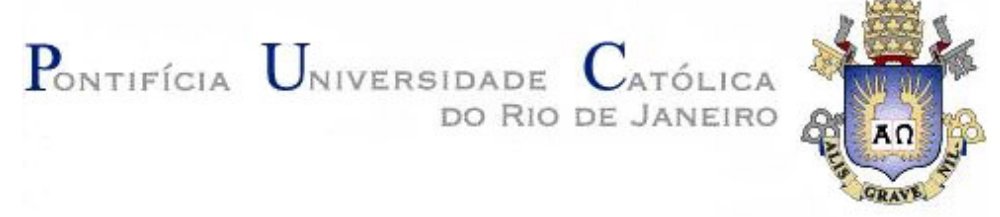

João Luiz de Oliveira Santa Rita

\title{
Estratégia de contratação de fretes: um estudo de caso na indústria do cimento
}

\begin{abstract}
Dissertação apresentada como requisito parcial para obtenção do grau de Mestre pelo Programa de Pós-Graduação em Engenharia de Produção do Departamento de Engenharia Industrial da PUC-Rio. Aprovada pela Comissão Examinadora abaixo assinada.
\end{abstract}

\author{
Prof. José Eugênio Leal \\ Orientador \\ Departamento de Engenharia Industrial - PUC-Rio
}

Profa. Adriana Leiras

Departamento de Engenharia Industrial - PUC-Rio

Prof. Hugo Repolho

Departamento de Engenharia Industrial - PUC-Rio

Prof. José Eugênio Leal

Coordenador Setorial do centro Técnico Científico - PUC-Rio

Rio de Janeiro, 02 de maio de 2013 
Todos os direitos reservados. É proibida a reprodução total ou parcial do trabalho sem autorização da universidade, do autor e do orientador.

\section{João Luiz de Oliveira Santa Rita}

Graduou-se em Administração de Empresas pela UNIGRANRIO em 2007, cursou pós-graduação em Auditoria e Controladoria pela Universidade Cândido Mendes entre 2008 e 2009 e MBA Executivo em Gestão Empresarial pela Politécnica da UFRJ entre 2008 e 2009. Atua, desde 1988, na área de Suprimentos de diversas empresas.

Ficha Catalográfica

Santa Rita, João Luiz de Oliveira

Estratégia de contratação de fretes: um estudo de caso na indústria do cimento / João Luiz de Oliveira Santa Rita; orientador: José Eugênio Leal. - 2013.

111 f.: il. (color.); $30 \mathrm{~cm}$

Dissertação (mestrado)-Pontifícia Universidade Católica do Rio de Janeiro, Departamento de Engenharia Industrial, 2013.

Inclui bibliografia

1. Engenharia Industrial - Teses. 2. Planejamento de transportes. 3. Compras estratégicas. 4. Contratação de fretes. I. Leal, José Eugenio. II. Pontifícia Universidade Católica do Rio de Janeiro. Departamento de Engenharia Industrial. III. Título.

CDD: 658.5 


\section{Agradecimentos}

À minha esposa Cláudia Cristina, por me amparar e auxiliar em todos os dias que me envolvi neste trabalho, e à minha filha Isabela, um anjo que traz luz e amor ao nosso lar.

Ao meu Orientador e agora Amigo Professor José Eugenio Leal, sempre solícito, disposto a ajudar e instruir, contribuindo para meu crescimento profissional e pessoal.

À minha Mãe Rosa, que sempre me apoiou e incentivou nos momentos difíceis. 


\section{Resumo}

Santa Rita, João Luiz de Oliveira; Leal, José Eugênio (Orientador). Estratégia de contratação de fretes: um estudo de caso na indústria do cimento. Rio de Janeiro, 2013. 111p. Dissertação de Mestrado (Opção profissional) - Departamento de Engenharia Industrial. Pontifícia Universidade Católica do Rio de Janeiro.

Compradores de todo o mundo estão cada vez mais informados e conscientes de como exigir dos vendedores que assumam maiores riscos, para ganhar o valorizado negócio do comprador. A integração de produtos, serviços e soluções crescem a uma taxa exponencial em transações comerciais. Assim, os contratos são cada vez mais complicados para planejar, negociar e administrar. Globalmente, as empresas têm aumentado exponencialmente o número de terceirizações para bens e serviços, com a intenção específica de se concentrar mais em seu negócio principal. Este estudo tem em vista ampliar os conhecimentos da gestão estratégica de transportes, com foco na contratação de fretes, analisando o relacionamento das organizações com seus fornecedores, integrados em um cenário de rápidas mudanças. Qualquer empresa deseja ser rápida e ter efetiva e contínua integração interna, com seus clientes e fornecedores, construindo, assim, parcerias duradouras e de sucesso. A vantagem competitiva que pode surgir a partir das estratégias de compra, é evidenciada, considerando-se os tipos de relações adotadas com os fornecedores. No estudo de caso, procurou-se mostrar a efetiva aplicação do planejamento estratégico no moderno gerenciamento da logística de transportes, importância do relacionamento com fornecedores, não somente na negociação de preços, prazos e qualidade, mas na adição do valor em toda a cadeia. A forma de gerenciamento, que uma organização pratica hoje, irá afetar significativamente seus resultados no mercado do futuro.

\section{Palavras-chave}

Planejamento de transporte; Compras estratégicas; Contratação de fretes. 


\section{Abstract}

Santa Rita, João Luiz de Oliveira; Leal, José Eugênio (Advisor). Strategy for freight contracting: a case study in the cement industry. Rio de Janeiro, 2013. 111p. MSc. Dissertation - Departamento de Engenharia Industrial. Pontifícia Universidade Católica do Rio de Janeiro.

Buyers worldwide are becoming more informed and demanding as they require sellers to assume greater business risks to win the buyer's valued business. The integration of products, services and solutions is growing at an exponential rate in business transactions. Thus, contracts are becoming more complicated to plan, negotiate and administer. Globally, companies have increased their contracts for goods and services at an exponential rate, with the specific intend of focusing more on their core business. The objective of this study is to broaden the knowledge about transport logistics strategy, focused on contracting freight, analyzing the relationship between companies and their suppliers, integrated into a scenario of rapid change. What every company wants is a fast, effective, and seamless integration between itself, each customer and its supply chain, building successful and long business partnerships. The competitive advantage that can arise from the purchase strategies has been evidenced considering the types of relations adopted with the suppliers. In the case study it was attempted to evidence the effective implementation of the modern transport logistics management actions, analyzing the evolution of the purchasing activity, giving emphasis to the relevance of the relation with suppliers not only negotiating price, terms and quality but also attaching higher value to the supply chain. Likewise, the contracts an organization negotiates today and performs tomorrow will significantly affect its profitability and reputation in the market place of the future.

\section{Keywords}

Transport Planning; Strategic Sourcing; Carrier Outsourcing. 


\section{Sumário}

1. Introdução 13

1.1. Definição do problema e delimitação 14

1.1. Objetivo geral 14

1.2. Objetivos específicos 14

1.3. Metodologia do trabalho 15

$\begin{array}{ll}\text { 1.4. Estrutura do trabalho } & 15\end{array}$

2. Revisão bibliográfica 17

2.1. Logística de transportes 17

2.2. Operador Logístico e suas atribuições 17

2.3. Transportador rodoviário de cargas 19

2.4. Administração de Materiais 20

2.5. Logística de distribuição 20

2.6. Planejamento estratégico 21

2.7. O processo de compra estratégica 23

3. Estudo de Caso 25

3.1. Caracterização da operação sudeste - outbound 27

3.1.2. Volumes por instalação - outbound 27

3.1.3. Fracionamento de entregas - outbound 29

3.1.4. Análises por faixa de distância - outbound 30

3.1.5. Análises por Perfil de Transportador - outbound 31

3.2. Caracterização da operação Sudeste - inbound 34

3.2.1. Volumes por Instalação - inbound 34

3.2.3. Análise da contratação - inbound 35

3.2.4. Análise do perfil de transportador - inbound 37

3.4. Diretrizes de Segurança 38

4. Análise do mercado de transporte rodoviário 39

4.1. Comportamento do Mercado - Transportadores 39

4.1.1. Análise financeira e de mercado 39

4.1.2. Percepção dos transportadores sobre o mercado 40 
4.2. Tendências 41

4.3. Comportamento do Mercado - Embarcadores 43

4.3.1. Gestão do transporte - embarcadores 43

4.3.2. Tendências de preço - embarcadores 46

4.4. Entrevistas $\quad 47$

4.4.1. Transportador 47

4.4.2. Embarcadores $\quad 49$

5. Mapeamento da oferta de transportes (RFI) 52

5.1. Motivação e responsividade do RFI 52

5.2. Caracterização da Amostra de Respondentes 52

5.3. Gestão e TI 56

5.4. Segurança e Qualidade 56

5.5. Contexto de mercado $\quad 57$

5.6. Desempenho das Transportadoras $\quad 59$

6. Modelo de custeio de frete 60

6.1. Ganhos de Eficiência com o aumento do nível de segurança 62

7. Definição da estratégia de transportes 64

7.1. Dados para definição das estratégias 64

$\begin{array}{ll}\text { 7.1.1. Contexto atual } & 65\end{array}$

7.1.2. Comportamento da operação Sudeste 66

7.2. Modelo de Contratação 66

7.2.1. Objetivos gerais do modelo 66

7.2.2. Contratação de fretes - regras e parâmetros 68

7.2.2.2. Rotas de Transferência $\quad 72$

$\begin{array}{ll}\text { 7.2.2.3. Granel } & 72\end{array}$

7.2.2.4. Inbound 72

7.2.2.5. Entregas locais $\quad 72$

$\begin{array}{ll}\text { 7.2.2.6. Resumo das regras e parâmetros } & 73\end{array}$

7.2.3. Modelo de Contratação - Visão Geral 76

$\begin{array}{ll}\text { 7.2.4 Transportadoras Potenciais } & 77\end{array}$

7.3. Aplicação do modelo - estratégias de contratação 78

7.3.1. Estratégia de contratação - inbound 78

$\begin{array}{ll}\text { 7.3.1.1. Parâmetros do inbound } & 79\end{array}$

7.3.1.2. Impactos em Custos - inbound 80 
7.3.2. Estratégia de Contratação - Corredores de Transferência 81

7.3.2.1. Parâmetros da Transferência 82

7.3.2.2. Impactos em Custos - Transferência 82

7.3.3. Estratégia de contratação - corredores de distribuição 83

7.3.3.1. Parâmetros da distribuição 84

7.3.3.2. Impactos em Custos - distribuição 84

7.3.3.3. Retorno pago - distribuição $\quad 85$

7.3.4. Estratégia de contratação - Entrega Local - CDs 86

7.3.4.1. Parâmetros da entrega local 86

7.3.4.2. Impactos em custos - entrega local 86

7.3.5. Estratégia de contratação - Granel 87

7.3.5.1. Parâmetros do Granel 89

7.3.5.2. Impactos em Custos - Granel 89

7.3.6. Modelo de contratação - custos Totais 90

7.3.7. Estratégias de contratação de fretes 91

$\begin{array}{ll}\text { 7.4. Implementação } & 91\end{array}$

8. Conclusões e recomendações futuras 93

8.1. Conclusões 93

8.2. Recomendações futuras $\quad 95$

9. Referencias Bibliográficas 96

10. Apêndices 99

10.1. Apêndice 1 - Ferramenta de Custeio 99

10.2. Apêndice 2 - Planilha de resposta do RFI (Request for Information) 101

10.3. Apêndice 3 - Planilha do esboço do plano de ação. 106

10.4. Apêndice 4 - Planilha de auditoria de contratados. 107

10.5. Apêndice 5 - Planilha fórmulas do custeio. 108

10.6. Apêndice 6 - Representatividade Sudeste 109

10.7. Apêndice7 - Classificação geral - RFI 111 


\section{Lista de figuras}

Figura 1 - Geração de valor à função Suprimentos 24

Figura 2 - Custos de transporte no Sudeste 26

Figura 3 - Valor e representatividade - custos no outbound 26

Figura 4 - Movimentação por município de destino (outbound - Sudeste) 27

Figura 5 - Outbound - \% na distribuição (venda) e transferência 28

Figura 6 - Volumes das fabricas - granel x ensacado 28

Figura 7 - Quantidade de viagens $x$ destinos e volume médio por viagem 29

Figura 8 - Fracionamento de entregas - viagens x destinos (CIF) 29

Figura 9 - Perfil geral - volume CIF com origem fábrica 30

Figura 10 - Perfil geral - distâncias das viagens fluxo CIF com origem fábrica 30

Figura 11 - Perfil geral - distâncias das viagens fluxo CIF com origem CDs 31

Figura 12 - Transportadoras x autônomos - transferência e distribuição 31

Figura 13 - Concentração de veículos - perfil geral 33

Figura 14 - Dispersão de preços do frete - Distribuição 34

Figura 15 - Volumes por instalação - inbound 35

Figura 16 - Inbound - planejado x não planejado e fluxos em volume e ativo 36

Figura 17 - Distâncias e contratação (volume) 36

Figura 18 - Distâncias e perfil de contratação inbound FOB 37

Figura 19 - Transporte planejado e não planejado - inbound 38

Figura 20 - Desempenho das Transportadoras em auditoria de segurança 38

Figura 21 - Receita e lucro operacional 39

Figura 22 - Atuação geográfica e indicadores $\quad 40$

Figura 23 - Diversificação de operações 40

Figura 24 - Atendimento da demanda 41

Figura 25 - Custo do transporte 43

Figura 26 - Descentralização do transporte 43

Figura 27 - Carga movimentada por responsável $\quad 44$

Figura 28 - Forma de seleção do transportador $\quad 44$

Figura 29 - Percentual de contratos formais $\quad 45$

Figura 30 - Gatilho do diesel $\quad 45$

Figura 31 - Exigência de idade da frota 46

Figura 32 - Principais indicadores de desempenho 46

$\begin{array}{ll}\text { Figura } 33 \text { - Grau de dificuldade na contratação } & 47\end{array}$ 
Figura 34 - Contratação de autônomos $\quad 48$

Figura 35 - Gestão do transporte $\quad 50$

Figura 36 - Tendência - frota dedicada x autônomos 51

Figura 37 - Faturamento e volume transportado - RFI 53

Figura 38 - Filiais e funcionários - RFI 53

Figura 39 - Representatividade setorial - RFI 54

Figura 40 - Especialização do transporte - RFI 54

Figura 41 - Quantidade de empresas por tipo de ativo de transporte - RFI 55

Figura 42 - Gestão de segurança e gestão de riscos - RFI 57

Figura 43 - Gestão dos motoristas - RFI $\quad 57$

Figura 44 - Grau de terceirização na operação 58

Figura 45 - Ativos - empresas x autônomos - RFI 58

Figura 46 - Custos básicos $\quad 60$

Figura 47 - Remuneração do frete fracionado 69

Figura 48 - Entrega fracionada - percentual do volume por distância 69

Figura 49 - Modelo de Contratação $\quad 76$

Figura 50 - Critérios de escolha das transportadoras potenciais $\quad 77$

Figura 51 - Dados das rotas inbound 78

Figura 52 - Rotas de caçamba, por tipo e padrão de comportamento 79

Figura 53 - Rotas de transferência $\quad 81$

Figura 54 - Concentração de entregas no granel 88

Figura 55 - As cinco estratégias de contratação 91

$\begin{array}{ll}\text { Figura } 56 \text { - Sequenciamento geral } & 91\end{array}$ 


\section{Lista de tabelas}

Tabela 1 - Curva ABC - volume e frete CIF outbound 32

Tabela 2 - Concentração de transportadoras - Granel 32

Tabela 3 - Curva ABC - volume e frete pago - inbound 37

Tabela 4 - Fluxos compatíveis - viagens e retorno - RFI 56

Tabela 5 - Regras e parâmetros gerais $\quad 75$

$\begin{array}{ll}\text { Tabela } 6 \text { - Parâmetros inbound } & 79\end{array}$

Tabela 7 - Metas de mix de frota e impactos no inbound 80

Tabela 8 - Parâmetros na transferência 82

Tabela 9 - Metas de mix de frota e impactos - transferência 83

Tabela 10 - Parâmetros da distribuição (ensacado) 84

Tabela 11 - Metas de mix de frota e impactos - distribuição 85

Tabela 12 - Estimativas de frete retorno - distribuição 85

Tabela 13 - Parâmetros da entrega local (ensacado) 86

Tabela 15 - Volume e variação - granel 88

Tabela 16 - Parâmetros no granel $\quad 89$

Tabela 17 - Metas de mix de frota e impactos - granel 90

Tabela 18 - Conta frete por estratégia e custo total 90 